\title{
GENETIC AND EPIGENETIC FACTORS CONTROLLING FEMALE STERILITY IN NEUROSPORA CRASSA
}

\author{
P. H. FITZGERALD \\ Cytogenetics Unit, Christchurch Hospital, Christchurch, New Zealand
}

\section{INTRODUCTION}

Received 20.vi.62

THE production of protoperithecia, the female reproductive structures, in Neurospora crassa is influenced by environmental and genetic factors. Westergaard and Mitchell (1947) developed a synthetic culture medium, which was optimal for protoperithecial formation and subsequent maturation of perithecia. A temperature of $25^{\circ} \mathrm{C}$. gave rapid and strong development of protoperithecia and perithecia, while a temperature of $35^{\circ} \mathrm{C}$. inhibited the formation of both, findings confirmed by Hirsch (1954). Knowledge of how to control protoperithecial production makes $\mathcal{N}$. crassa particularly suitable for genetic studies of their formation. Genetic factors influencing the development of protoperithecia have been recorded in Neurospora and other ascomycetes. Dodge (1946) found that lack of protoperithecia in $\mathcal{N}$. sitophila was caused by a single gene. Protoperithecial abnormalities and lack of protoperithecia in strains of $\mathcal{N}$. crassa with long histories of vegetative propagation, found by Westergaard and Hirsch (1954), appeared to be caused by different, non-allelic, mutant genes. Jinks (I954, I956) demonstrated a cytoplasmic basis for reduced and permanent loss of perithecial formation in the homothallic Aspergillus nidulans and $A$. glaucus after continued asexual propagation by conidia and after ageing.

Failure to develop protoperithecia in a strain of Neurospora crassa is shown in this paper to be caused by the joint action of genetic factors and a cellular control system having an epigenetic action (Nanney, I958).**

The strain of $\mathcal{N}$. crassa concerned, originated from an Emerson $A$ $\times$ Emerson $a$ cross, was prototrophic and of mating type $A$. It showed normal fertility during several years of vegetative propagation, but protoperithecial production subsequently weakened, and eventually, all isolations failed to develop protoperithecia. Subcultures of these isolations likewise did not form protoperithecia. Crosses with other normal strains were completely sterile when these isolations were used as female parents, but normal perithecia and ascospores resulted when they were used as male parents.

\section{METHODS}

Prototrophic strains were involved throughout the investigation, and these were cultured at $25^{\circ} \mathrm{C}$. on the minimal medium of Westergaard and Mitchell (1947), which promotes protoperithecial production.

* The use of the term " epigenetic ", and alternatives, is discussed by Catcheside (1959). 
In the serial subcultures, inocula from a number of stock lines were incubated at $35^{\circ} \mathrm{C}$. for rapid growth on minimal agar slopes, and after 24 hours, hyphal tips were removed and used to inoculate fresh slopes. These were again incubated at $35^{\circ} \mathrm{C}$. for 24 hours and the subculture repeated successively. After removal of the hyphal tips, the cultures were incubated at $25^{\circ} \mathrm{C}$. for two or more weeks. This allowed protoperithecial development, and any phenotypic differences at successive stages of the treatment could be detected. Some cultures were incubated completely at $35^{\circ} \mathrm{C}$. This treatment allowed rapid growth of young hyphæ for several successive 24 hour periods, and created a series of discontinuities between the newly formed hyphæ and possible influences of the old hyphæ.

\section{GENETICS OF THE FEMALE STERILITY}

\section{(i) The wild-type $\times$ female sterile progeny}

Eighteen asci were isolated from a cross between wild-type and female-sterile strains. Four of the asci, from two perithecia, contained cultures with abnormal growth or melanin production and were not

TABLE 1

Progenies of the crosses : wild-type $\times$ female-sterile, and small $\times$ breakdown.

\begin{tabular}{|c|c|c|c|c|c|c|c|c|c|}
\hline Parents & \multicolumn{8}{|c|}{ Ascus types in progeny } & \multirow{2}{*}{$\begin{array}{c}\begin{array}{c}\text { Numbers } \\
\text { of asci }\end{array} \\
\\
4 \\
3 \\
4 \\
1 \\
2 \\
4\end{array}$} \\
\hline $\begin{array}{l}\text { Wild type } a \times \\
\text { female-sterile A }\end{array}$ & $\begin{array}{l}\mathrm{La} \\
\mathrm{La} \\
\mathrm{La} \\
\mathrm{La} \\
\mathrm{La}\end{array}$ & $\begin{array}{l}\mathrm{La} \\
\mathrm{La} \\
\mathrm{La} \\
\mathrm{La} \\
\mathrm{La}\end{array}$ & $\begin{array}{l}\mathrm{La} \\
\mathrm{La} \\
\mathrm{La} \\
\mathrm{La} \\
\mathrm{La}\end{array}$ & $\begin{array}{l}\mathrm{La} \\
\mathrm{La} \\
\mathrm{La} \\
\mathrm{La} \\
\mathrm{La} \\
\mathrm{Abn}\end{array}$ & \begin{tabular}{|}
$\left|\begin{array}{c}\mathrm{Sm} \\
\mathrm{Sm} \\
\mathrm{St} \\
\mathrm{St} \\
\mathrm{St}\end{array}\right|$ \\
ormal asc
\end{tabular} & $\begin{array}{l}\mathrm{Sm} \\
\mathrm{Sm} \\
\mathrm{St} \\
\mathrm{Sm} \\
\mathrm{St} \\
\end{array}$ & $\begin{array}{l}\mathrm{Sm} \\
\mathrm{Bk} \\
\mathrm{Bk} \\
\mathrm{Bk} \\
\mathrm{Sm}\end{array}$ & $\begin{array}{l}\mathrm{Sm} \\
\mathrm{Bk} \\
\mathrm{Bk} \\
\mathrm{Bk} \\
\mathrm{Sm}\end{array}$ & \\
\hline $\begin{array}{l}3-6 \mathrm{~A} \times 14-8 a \\
\text { small } \times \text { breakdown }\end{array}$ & $\begin{array}{l}\text { Sm } \\
\text { Sm } \\
\text { Sm } \\
\text { Sm }\end{array}$ & $\begin{array}{l}\mathrm{Sm} \\
\mathrm{Sm} \\
\mathrm{Sm} \\
\mathrm{Sm}\end{array}$ & $\begin{array}{l}\mathrm{Bk} \\
\mathrm{Bk} \\
\mathrm{Sm} \\
\mathrm{Sm}\end{array}$ & $\begin{array}{c}\mathrm{Bk} \\
\mathrm{Bk} / \mathrm{Sm} \\
\mathrm{Sm} \\
\mathrm{Sm}\end{array}$ & $\begin{array}{c}\mathrm{Bk} / \mathrm{Sm} \\
\mathrm{Sm} \\
\mathrm{Bk} / \mathrm{Sm} \\
\mathrm{Sm}\end{array}$ & $\begin{array}{l}\mathrm{Bk} \\
\mathrm{Sm} \\
\mathrm{Bk} \\
\mathrm{Sm}\end{array}$ & $\begin{array}{c}\mathrm{Sm} \\
\mathrm{Bk} / \mathrm{Sm} \\
\mathrm{Bk} / \mathrm{Sm} \\
\mathrm{Bk}\end{array}$ & $\begin{array}{c}\mathrm{Sm} \\
\mathrm{Bk} / \mathrm{Sm} \\
\mathrm{Sm} \\
\mathrm{Bk}\end{array}$ & $\begin{array}{l}3 \\
1 \\
1 \\
4\end{array}$ \\
\hline Ascus 16 & $\mathrm{Sm}$ & $\mathrm{Sm}$ & $\mathrm{Bk}$ & $\mathrm{Bk}$ & $\mathrm{Bk} / \mathrm{Sm}$ & $\mathrm{Bk}$ & $\mathrm{Sm}$ & Sm & \\
\hline $\begin{array}{l}16-1 A \times 16-6 a \\
16-8 a \times 16-3 A\end{array}$ & $\begin{array}{l}\overline{S m} \\
\text { Sm }\end{array}$ & $\begin{array}{l}\mathrm{Sm} \\
\mathrm{Sm} \\
\mathrm{Sm}\end{array}$ & $\begin{array}{l}\mathrm{Sm} \\
\mathrm{Bk} \\
\mathrm{Sm}\end{array}$ & $\begin{array}{l}\mathrm{Sm} \\
\mathrm{Bk} \\
\mathrm{Sm}\end{array}$ & $\begin{array}{l}\mathrm{Bk} \\
\mathrm{Bk} \\
\mathrm{Sm}\end{array}$ & $\begin{array}{l}\mathrm{Bk} \\
\mathrm{Bk} \\
\mathrm{Sm}\end{array}$ & $\begin{array}{l}\mathrm{Sm} \\
\mathrm{Sm} \\
\mathrm{Bk}\end{array}$ & $\begin{array}{l}\text { Sm } \\
\text { Sm } \\
-\end{array}$ & $\begin{array}{l}1 \\
2 \\
1\end{array}$ \\
\hline
\end{tabular}

Phenotypes are indicated as follows :- La, large; $\mathrm{Sm}$, small; $\mathrm{St}$, sterile; $\mathrm{Bk}$, breakdown.

scored. The remaining fourteen asci, from six other perithecia, contained four phenotypes as follows: the normal wild-type with large protoperithecia covering most of the culture surface (phenotype large); small, pale-coloured yet functional protoperithecia mostly confined to the mycelium at the edges of the culture medium (phenotype small); a female-sterile strain without protoperithecia (phenotype sterile); a female-sterile strain producing many small, discrete, dark-brown 
coloured patches on and in the agar medium at the time protoperithecia are normally differentiated, and accompanied, as are protoperithecia, by a general darkening of the culture medium due to melanin formation. Microscopic examination showed that the dark-brown patches had a fine granular structure, and occurred where cell contents escaped through ruptures in the walls of enlarged hyphal cells. Cells swollen with dark-brown granular contents but as yet unbroken also occurred. As these dark patches appeared to result from a breakdown in the normal differentiation of protoperithecia, this phenotype was called breakdown.

The patterns of these four phenotypes in the asci, and the frequencies of different ascus types are shown in table $\mathrm{I}$. Large and non-large spore pairs segregated at the first division in all asci. One spore pair showed both phenotypes small and sterile. All four phenotypes were obtained from single perithecia, and did not therefore result from heterocaryosis in the parent strains.

When subcultured on the same medium, large strains produced protoperithecia by the sixth day, and small subcultures produced their characteristic protoperithecia in ten to sixteen days. Subcultures of the sterile strains likewise produced protoperithecia at this time and could not be distinguished from the small phenotype, while subcultures of breakdown strains did not form dark-coloured centres of hyphal breakdown, and instead developed a few small protoperithecia. The change of phenotypes sterile and breakdown to small was persistent in serial subcultures, and was also observed in nearly all subcultures of sterile and breakdown strains in later progenies. Intercrosses of the progeny strains are considered in the following sections.

(ii) Crosses between small and breakdown strains

Six small strains were crossed by subcultures of breakdown strains which had changed to the small phenotype, and the progeny of one of these crosses 3-6 $A \times 14-8 a$ is shown in table $\mathrm{I}$. Breakdown reappeared in the progenies. Some breakdown cultures also produced small protoperithecia and showed both phenotypes at the same time. These cultures occurred in spore pairs with either breakdown or small cultures, and appeared to be an earlier manifestation of the change to small observed previously in subcultures of breakdown strains. The progeny contained, in general, asci with 3 small and I breakdown spore pairs, and asci with 2 small and 2 breakdown spore pairs. The cultures showing both breakdown and small occurred only in the latter asci. Subcultures of the breakdown ascospore cultures showed no sign of breakdown and developed small protoperithecia or, in a few cases, no protoperithecia. Reciprocal crosses using breakdown subcultures as female parents gave no progeny, probably because of their imperfectly developed protoperithecia.

The small and breakdown strains from one of the 2 small : 2 breakdown asci (ascus no. I6) were intercrossed, and the progenies (table I), 
although small because of poor ascospore germination, showed 2 small: 2 breakdown asci and 3 small : I breakdown asci, and so were similar to the progenies already described. Subcultures of the breakdown cultures in these progenies all developed phenotype small.

These results suggested that more than one gene distinguished small and breakdown. The presence of two or more major genes was tested by analysing three asci containing 3 small and I breakdown spore pairs. These three analyses gave similar results and one of them (ascus no. 2I) is shown in table 2. Because of poor ascus maturity in these and previous crosses, the progenies were obtained from random ascospores. The high germination percentages in many of these

TABLE 2

Analysis of ascus $2 I$ (random spore progenies)

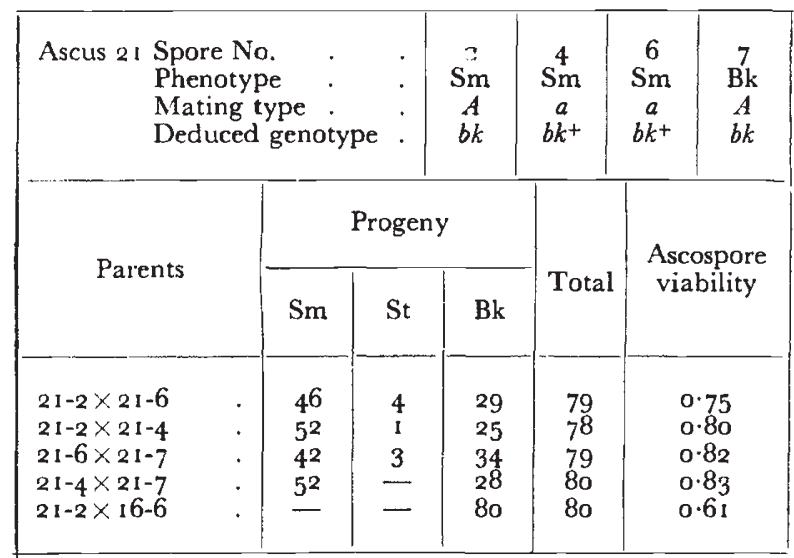

progenies suggested that the ascospores ripened after discharge from the perithecia. The three small strains in ascus 2 I were intercrossed as their mating types allowed, and all three were crossed by a breakdown strain. Because of mating type differences, small strain 2I-2A was crossed by breakdown strain I6-6a from another ascus. Crosses 2 I-2 $A$ $\times 2 \mathrm{I}-6 a$ and $2 \mathrm{I}-2 A \times 2 \mathrm{I}-4 a$ involved small strains only, yet the progenies of both contained breakdown cultures. This result was impossible if two or more genes determined small, when at least one of the crosses would have given an all small progeny, and indicated a single gene difference. The strain common to both crosses 2 I-2 $A$ presumably did not carry the gene determining small, as is further shown by the lack of small segregants from the small $\times$ breakdown cross 2 I-2 $A \times 16-6 a$. This progeny was the same as would be expected from a breakdown $\times$ breakdown cross, and indicated that $2 \mathrm{I}-2 A$, although phenotypically small, had the same genotype as breakdown strains, that is it carried a mutant gene $b k$. Likewise, $2 \mathrm{I}-4 a$ and $2 \mathrm{I}-6 a$ carried a factor $b k^{+}$, determining phenotype small. The progenies of the first four crosses analysing ascus 2 I varied little from each other ; and the deviation from the I 
small: I breakdown ratio expected from a one gene difference was similar to that in the original small $\times$ breakdown progeny (table I) where it was caused by some $b k$ strains showing phenotype small. It was found that such pseudo-small strains could be distinguished from true small strains by their later development of protoperithecia and by a general darkening of the medium as they aged, as was characteristic of breakdown strains. When such strains were taken into account, the random ascospore progenies showed good I: I ratios. The genotypes of fifteen of these pseudo-small strains were checked by crossing them to strains known to carry $b k^{+}$. All gave progenies containing breakdown cultures, whereas four small strains, which did not darken with ageing and showed an earlier development of protoperithecia, gave progenies of small cultures only.

All of the small $\times$ breakdown crosses involved breakdown strains which had developed phenotype small as a result of being subcultured. The results therefore showed that the change from breakdown to small did not involve a gene change. Indeed, the phenotypic expression of allele $b k$ was most variable, and the ease with which it could be changed, as for example by subculture, and the occurrence of different phenotypes in the same spore pair, both indicated that it was largely controlled by environmental or other non-genetic factors.

\section{(iii) Crosses between large and breakdown strains}

Two types of progenies resulted from crosses between large strains and breakdown strains which had changed to the small phenotype. The first type, obtained from eight of the large strains, contained only asci with a clear segregation of two large spore pairs and two spore pairs showing phenotypes breakdown, pseudo-small and sterile. The progeny of one of these crosses $10-3 a \times 6-8 A$ is shown in table $3(a)$. Seventeen of the pseudo-small and sterile cultures from different progenies were crossed to true small tester stocks and all produced some breakdown progeny. One gene determined phenotype large in these asci and $b k$ showed variable phenotypic expression, including differences within spore pairs, as in the small and breakdown progenies.

In the second type of progeny, which resulted from four of the large strains, the asci likewise contained two large spore pairs, whereas the other two spore pairs showed the true small phenotype as well as breakdown, pseudo-small and sterile. The progeny of cross 6-4a 6 6-8A which was of this type is shown in table $3(b)$. The true small strains gave progenies of small cultures only, when crossed to tester strains. Allele $b k^{+}$determining phenotype small was segregating in this progeny, and the parental strains differed by two genes.

Large strains were of two genetic types: those differing from breakdown by one gene $s^{+}$determining large, and those differing from breakdown by genes $s^{+}$and $b k^{+}$. Genotypes $s^{+} b k^{+}$and $s^{+} b k$ determined phenotype large, $s b k+$ determined small, and $s b k$ produced phenotypes 
ranging from breakdown through intermediate sterile forms to pseudosmall according to the degree of its expression. The equal segregation of large and non-large spore pairs in all asci showed that $s^{+}$was epistatic to the alleles at the $b k$ locus. Random spore progenies from crosses of the twelve large strains by breakdown gave similar results to these.

Seven of the large $\left(s^{+} b k\right) \times$ breakdown crosses were repeated from cultures of parental strains which had been subjected to serial subculture under the conditions, described in section 4, leading to the

TABLE 3

Progenies of large $\times$ breakdown crosses

\begin{tabular}{|c|c|c|c|c|c|c|c|c|c|c|}
\hline \multirow[b]{2}{*}{$a$} & Parents & \multicolumn{8}{|c|}{ Ascus types in progeny } & $\begin{array}{l}\text { Numbers } \\
\text { of Asci }\end{array}$ \\
\hline & $10-3 a \times 6-8 A$ & $\begin{array}{l}\mathrm{La} \\
\mathrm{La} \\
\mathrm{La} \\
\mathrm{La} \\
\mathrm{La} \\
\mathrm{La}\end{array}$ & $\begin{array}{l}\mathrm{La} \\
\mathrm{La} \\
\mathrm{La} \\
\mathrm{La} \\
\mathrm{La} \\
\mathrm{La}\end{array}$ & $\begin{array}{l}\mathrm{La} \\
\mathrm{La} \\
\mathrm{La} \\
\mathrm{La} \\
\mathrm{La} \\
\mathrm{La}\end{array}$ & $\begin{array}{l}\mathrm{La} \\
\mathrm{La} \\
\mathrm{La} \\
\mathrm{La} \\
\mathrm{La} \\
\mathrm{La}\end{array}$ & $\begin{array}{l}\mathrm{Sm}^{\prime} \\
\mathrm{Sm}^{\prime} \\
\mathrm{Sm}^{\prime} \\
\mathrm{Sm}^{\prime} \\
\mathrm{St} \\
\mathrm{Bk}\end{array}$ & $\begin{array}{c}\mathrm{Sm}^{\prime} \\
\mathrm{St} \\
\mathrm{Bk} \\
\mathrm{Sm}^{\prime} \\
\mathrm{St} \\
\mathrm{Bk}\end{array}$ & $\begin{array}{c}\mathrm{Sm}^{\prime} \\
\mathrm{Sm}^{\prime} \\
\mathrm{Sm}^{\prime} \\
\mathrm{Bk} \\
\mathrm{Bk} \\
\mathrm{Bk}\end{array}$ & $\begin{array}{c}\mathrm{Sm}^{\prime} \\
\mathrm{Sm}^{\prime} \\
\mathrm{Sm}^{\prime} \\
\mathrm{Bk} \\
\mathrm{Bk} \\
\mathrm{Bk}\end{array}$ & $\begin{array}{l}2 \\
1 \\
2 \\
2 \\
1 \\
2\end{array}$ \\
\hline$b$ & $6-4 a \times 6-8 A$ & $\begin{array}{l}\mathrm{La} \\
\mathrm{La} \\
\mathrm{La} \\
\mathrm{La} \\
\mathrm{La} \\
\mathrm{La}\end{array}$ & $\begin{array}{l}\mathrm{La} \\
\mathrm{La} \\
\mathrm{La} \\
\mathrm{La} \\
\mathrm{La} \\
\mathrm{La}\end{array}$ & $\begin{array}{l}\mathrm{La} \\
\mathrm{La} \\
\mathrm{La} \\
\mathrm{La} \\
\mathrm{La} \\
\mathrm{La}\end{array}$ & $\begin{array}{l}\mathrm{La} \\
\mathrm{La} \\
\mathrm{La} \\
\mathrm{La} \\
\mathrm{La} \\
\mathrm{La}\end{array}$ & $\begin{array}{c}\mathrm{Sm} \\
\mathrm{Sm} \\
\mathrm{Sm}^{\prime} \\
\mathrm{St} \\
\mathrm{St} \\
\mathrm{Bk}\end{array}$ & $\begin{array}{c}\mathrm{Sm} \\
\mathrm{Sm} \\
\mathrm{Sm}^{\prime} \\
\mathrm{St} \\
\mathrm{St} \\
\mathrm{Bk}\end{array}$ & $\begin{array}{l}\mathrm{Sm} \\
\mathrm{Sm}^{\prime} \\
\mathrm{Sm}^{\prime} \\
\mathrm{Sm}^{\prime} \\
\mathrm{St} \\
\mathrm{Sm}\end{array}$ & $\begin{array}{l}\mathrm{Sm} \\
\mathrm{Sm}^{\prime} \\
\mathrm{Sm}^{\prime} \\
\mathrm{Sm}^{\prime} \\
\mathrm{St} \\
\mathrm{Sm}\end{array}$ & $\begin{array}{l}\mathrm{I} \\
\mathrm{I} \\
4 \\
2 \\
\mathrm{I} \\
2\end{array}$ \\
\hline$c$ & $\begin{array}{c}\text { ro-3a } a \times 6-8 A \\
\text { (after serial } \\
\text { subculture) }\end{array}$ & $\begin{array}{l}\mathrm{La} \\
\mathrm{La} \\
\mathrm{La} \\
\mathrm{La}\end{array}$ & $\begin{array}{l}\mathrm{La} \\
\mathrm{La} \\
\mathrm{La} \\
\mathrm{La}\end{array}$ & $\begin{array}{l}\mathrm{La} \\
\mathrm{La} \\
\mathrm{La} \\
\mathrm{La}\end{array}$ & $\begin{array}{l}\mathrm{La} \\
\mathrm{La} \\
\mathrm{Lil} \\
\mathrm{La}\end{array}$ & $\begin{array}{l}\mathrm{Bk} \\
\mathrm{Bk} \\
\mathrm{Bk} \\
\mathrm{Bk}\end{array}$ & $\begin{array}{l}\mathrm{Bk} \\
\mathrm{Bk} \\
\frac{\mathrm{Bk}}{}\end{array}$ & $\begin{array}{l}\mathrm{Bk} \\
\mathrm{Bk} \\
\mathrm{Bk} \\
-\end{array}$ & $\begin{array}{l}\text { Bk } \\
\text { 二 }\end{array}$ & $\begin{array}{l}\mathrm{I} \\
5 \\
4 \\
\mathrm{I}\end{array}$ \\
\hline
\end{tabular}

Phenotypes are as indicated in earlier tables; also $\mathrm{Sm}^{\prime}$ denotes pseudo-small, and a dash denotes an inviable ascospore. First and second division segregation differences are not shown.

induction of breakdown in subcultures of $s b k$ strains. A marked effect on the expression of $s b k$ was observed in the progenies. The asci in these showed large and breakdown cultures only, with considerable ascospore inviability among the $s b k$ segregants. The progeny of cross $10-3 a \times 6-8 A$ (table $3(c)$ ) can be compared with the progeny of this cross already described in table $3(a)$. Only 2 of the $44 s^{+} b k$ ascospores were inviable compared with I 5 of the $44 s b k$ ascospores. Progenies of the other crosses showed near complete viabilities of $s^{+} b k$ segregants coupled with viabilities of $s b k$ segregants ranging from 4 to 50 per cent. Many spore pairs contained one inviable ascospore and one breakdown culture. The numbers of $s b k$ spore pairs containing 2, $\mathrm{I}$ and o viable breakdown ascospores agreed, in each progeny (probabilities ranging from 5 per cent. to go per cent.), with the numbers expected if inviability was distributed randomly in the population of breakdown ascospores and was not influenced by the pairs of genetically identical ascospores. 
The two series of large $\times$ breakdown crosses showed that a deficiency of breakdown in a progeny could result from inviability of $s b k$ ascospores and from incomplete expression of this genotype giving pseudo-small cultures. The occurrence of both these expressions of $s b k$ in progenies from different crossings of the same parental strains, differing only in pretreatment, indicated that they were epigenetic rather than genetic effects.

(iv) Genetic relationship of large, small and sterile strains

Random ascospore progenies from three crosses between small and sterile strains are shown in table 4 . The progeny of cross $3-6 A \times 5-6 a$

TABLE 4

Parent strains and random ascospore progenies of crosses between large, small and sterile strains

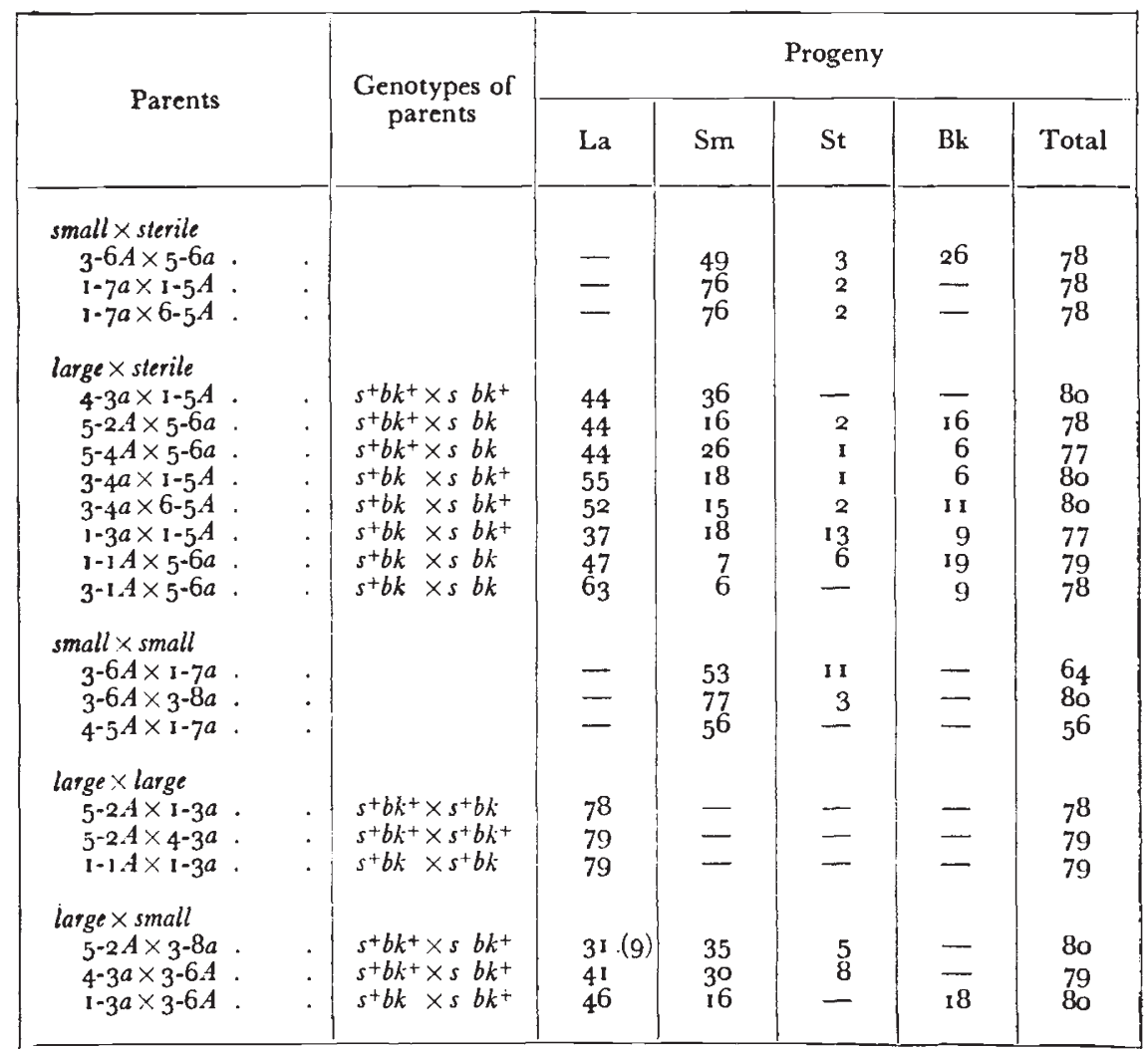

was the same as the random spore progenies obtained from small $\times$ breakdown crosses. Of six asci also isolated from this cross, three contained 3 small and 1 breakdown spore pairs and three contained 4 small spore pairs. Crosses to small tester stocks revealed that, in the latter asci, two of the small spore pairs were $s b k^{+}$whereas the other two segregated breakdown strains and were genetically $s b k$. The identity of these results to those of the small $\times$ breakdown crosses indicated that 
the sterile strain 5-6a was genetically $s b k$. The progenies of the other two small $\times$ sterile crosses consisted almost entirely of small cultures, and were the same as obtained from small $\times$ small crosses, indicating that the sterile strains $\mathrm{I}_{-5} A$ and $6-5 A$ were genetically $s b k^{+}$. The sterile phenotype did not represent a distinct genotype, but resulted from either failure to produce breakdown in a $s b k$ strains, or from failure to develop protoperithecia in $s b k^{+}$strains.

The parent strains and progenies from eight large $\times$ sterile crosses are presented in table 4 . The progenies showed a good correlation with the parental genotypes-as indicated by the results of previous crosses-after allowances were made for deficiencies of breakdown. These appeared to be due largely to inviability of breakdown ascospores in crosses $5-4 A \times 5-6 a, 3-4 a \times 1-5 A, 3-4 a \times 6-5 A$; and to reduced expression of $s b k$-appearing as sterile-in $\mathrm{I}-3 a \times \mathrm{I}-5 A$. The presence of some small cultures in the two $s^{+} b k \times s b k$ progenies, where an equal segregation of large and breakdown was expected, suggested reduced expression of $s b k$ although true and pseudo-small strains were not distinguished in these progenies. The results of these crosses in general agreed with the conclusion that sterile strains were genetically either $s b k^{+}$or $s b k$.

Small cultures were predominant in the progenies from three intercrosses of small strains (table 4) from the progeny of the original wild-type $\times$ female-sterile cross. Some sterile strains, presumably the result of failure to develop protoperithecia, were also present. These progenies agree with the earlier findings that phenotype small was determined by genotype $s b k^{+}$.

Progenies from three intercrosses of large strains (table 4) all contained large cultures only. The crosses represented all three combinations of the two large genotypes, and their results supported the earlier findings that $s^{+}$, determining phenotype large, was epistatic to the two alleles at the $b k$ locus.

The progenies of the first two large $\times$ small crosses (table 4) conformed in general to the equal segregation of large and small expected from their genotypes, although the expression of the segregating genotypes was not always clear. The sterile cultures were probably $s b k^{+}$in which full protoperithecial development was not realised. Nine cultures in the first progeny showed characters of both large and small phenotypes, and could not be classified into either group. The progeny of the third cross contained large, small and breakdown cultures in a ratio approximating $2: \mathrm{I}: \mathrm{I}$, which confirmed that the large and small phenotypes were determined by non-allelic factors, and that $s^{+}$ was epistatic to the $b k$ locus.

This analysis of the genetic relationship of the female-sterile and wild-type strains showed that two different genes determined protoperithecial formation, and that the expression of the double mutant genotype was subject to non-genetic variation. The genetic constitution of the six asci from the original wild-type $\times$ female-sterile progeny 
which were used in the analysis are shown in table 5. Asci I, 3 and Io showed one ditype segregation, ascus 5 the other ditype segregation, and asci 4 and 6 showed the tetratype segregation. Alleles $s^{+} / s$ and $b k^{+} / b k$ segregated at the first division in 89 per cent. (of I I 4 asci) and 39 per cent. (of 14 asci) respectively. The two loci were not likely to be linked to each other, and there was no evidence of linkage in

TABLE 5

Phenotypes and genotypes of asci $1,3,4,5,6$ and Io from the progeny of the original wild-type $\times$ female-sterile cross

\begin{tabular}{|c|c|c|c|c|}
\hline Ascus no. & Ascospore no. & Phenotype & Genotype & Mating type \\
\hline 1 & $\begin{array}{l}I-I \\
I-3 \\
I-5 \\
I-7\end{array}$ & $\begin{array}{l}\text { large } \\
\text { large } \\
\text { sterile } \\
\text { small }\end{array}$ & 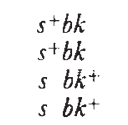 & $\begin{array}{l}\mathrm{A} \\
\mathrm{a} \\
\mathrm{A} \\
\mathrm{a}\end{array}$ \\
\hline 3 & $\begin{array}{c}3-1 \\
3-4 \\
3-6 \\
3-8\end{array}$ & $\begin{array}{l}\text { large } \\
\text { large } \\
\text { small } \\
\text { small }\end{array}$ & $\begin{array}{l}s^{+} b k \\
s^{+} b k \\
s \quad b k^{+} \\
s \quad b k^{+}\end{array}$ & $\begin{array}{l}\mathrm{A} \\
\mathrm{a} \\
\mathrm{A} \\
\mathrm{a}\end{array}$ \\
\hline סו & $\begin{array}{l}10-1 \\
10-3 \\
10-5 \\
10-7\end{array}$ & $\begin{array}{l}\text { large } \\
\text { large } \\
\text { small } \\
\text { small }\end{array}$ & $\begin{array}{l}s^{+} b k \\
s^{+} b k \\
s \quad b k^{+} \\
s \quad b k^{+}\end{array}$ & $\begin{array}{l}\mathrm{a} \\
\mathrm{a} \\
\mathrm{A} \\
\mathrm{A}\end{array}$ \\
\hline 4 & $\begin{array}{l}4-1 \\
4-3 \\
4-5 \\
4-8\end{array}$ & $\begin{array}{l}\text { large } \\
\text { large } \\
\text { small } \\
\text { breakdown }\end{array}$ & $\begin{array}{l}s^{+} b k \\
s^{+} b k^{+} \\
s \quad b k^{+} \\
s \quad b k\end{array}$ & $\begin{array}{l}\mathrm{a} \\
\mathrm{a} \\
\mathrm{A} \\
\mathrm{A}\end{array}$ \\
\hline 6 & $\begin{array}{l}6-2 \\
6-4 \\
6-5 \\
6-8\end{array}$ & $\begin{array}{l}\text { large } \\
\text { large } \\
\text { sterile } \\
\text { breakdown }\end{array}$ & $\begin{array}{l}s^{+} b k \\
s^{+} b k^{+} \\
s \quad b k^{+} \\
s \quad b k\end{array}$ & $\begin{array}{l}\mathrm{a} \\
\mathrm{a} \\
\mathrm{A} \\
\mathrm{A}\end{array}$ \\
\hline 5 & $\begin{array}{l}5-2 \\
5-4 \\
5-6 \\
5-7\end{array}$ & $\begin{array}{l}\text { large } \\
\text { large } \\
\text { sterile } \\
\text { breakdown }\end{array}$ & $\begin{array}{l}s^{+} b k^{+} \\
s^{+} b k^{+} \\
s \quad b k \\
s \quad b k\end{array}$ & $\begin{array}{l}\text { A } \\
\text { A } \\
\mathrm{a} \\
\mathrm{a}\end{array}$ \\
\hline
\end{tabular}

crosses where it would be detected. While these two genes account for the different types of protoperithecial formation in the progeny strain, the results of the wild-type $\times$ female-sterile cross were unusual in that the stable sterility which characterised the female-sterile parent was not present in the progeny.

\section{SERIAL SUBCULTURE}

(i) Phenotypic expression of $s$ bk

Serial subculture, details of which are given in section 2, was maintained for twenty successive subculture generations on twelve $s b k$ strains showing the small and sterile phenotypes. The breakdown phenotype reappeared in all of the strains, usually in the first generations 
treated, and, after a number of subculture generations which varied between different strains, it increased in intensity, showing a much greater production of the brown pigment. The appearance of breakdown was accompanied by a reduced growth rate, and as it intensified the growth rate dropped further. Two strains, in which breakdown was strongly developed, showed no growth at all during the first 24 hours of subculture generations 12 and 14 respectively, and only after this time was slow hyphal growth observed. In four other cases, involving three strains, inocula from cultures showing intense brown pigmentation and decreased growth rate were inviable. Intensification of breakdown reduced the viability of hyphæ.

The marked reappearance of breakdown in these treated strains contrasted with the results of previous continuous incubation at $35^{\circ} \mathrm{C}$. of four of them. Only two showed breakdown, and it did not persist in successive subcultures at $35^{\circ} \mathrm{C}$.

Subcultures of the twelve strains were taken from generations I 8 and 20 of the series by a mass inoculum of conidia and hyphæ, and incubated continuously at $25^{\circ} \mathrm{C}$. for about 30 days when they in turn were subcultured. This was repeated for five subculture generations. Under these normal culture conditions, the twelve strains mostly retained the abnormal development of breakdown induced by the serial subculture treatment. Four of the strains showed no indication of losing breakdown, but in some of the others its intensity was reduced. Prior to this treatment, breakdown was rarely expressed in subcultures incubated at $25^{\circ} \mathrm{C}$., but was an unstable condition found only in ascospore cultures, from which subcultures developed phenotype small.

The increased intensity of expression of breakdown after several treated generations, and its maintenance in some strains, for several subculture generations after the return to normal culture conditions showed that the changes in expression of $s b k$ from breakdown to small and from small to breakdown were not direct environmental effects. Rather it would appear that a physiological or cytoplasmic system, intervening between genotype $s b k$ and its phenotype, could exist in different states, some of which were self-perpetuating to some extent, and that these different states led to the different phenotypic expressions of $s b k$.

\section{(ii) Expression of the female-sterile strain}

The serial subculture treatment was maintained for ten successive subculture generations on the parent strains, and related strains, from the original wild-type $\times$ female-sterile cross, and on twelve large progeny strains. The phenotypes developed by successive stages of these strains showed the following characters. (a) Two separate lines of the female-sterile parent both developed breakdown strongly in the first treated stages, as did $s b k$ strains in the earlier series. However, in later stages of the treatment, breakdown disappeared and large protoperithecia developed. These persisted in five further subculture generations under normal culture conditions. (b) Breakdown did not appear in 
a female-fertile strain isolated asexually from the wild-type $A$ stock before it became female-sterile. (c) Breakdown did not appear in any of six separate lines of the wild-type $a$ stock used in the original cross. (d) None of the twelve large strains showed breakdown in cultures incubated at $25^{\circ} \mathrm{C}$. after the first 24 hours at $35^{\circ} \mathrm{C}$., but eight of them showed breakdown in cultures incubated at $35^{\circ} \mathrm{C}$. after this time. These eight strains were of genotype $s^{+} b k$, while the four strains which did not show breakdown were $s^{+} b k^{+}$. The conditions of the serial subculture treatment allowed the expression of allele $b k$ in these large cultures.

This behaviour of the large strains indicates that breakdown did not appear in the cultures of the wild-type $a$ parent of the original cross because this stock did not carry $b k$. This allele was introduced into the wild-type $\times$ female-sterile progeny by the female-sterile parent in which breakdown was well developed. Its absence in a female-fertile strain derived earlier from the same stock as the sterile strain suggested that development of the sterility was associated with the appearance of breakdown, and hence with the mutant $b k$.

(iii) Crosses involving the female-sterile strain

Cultures of the female-sterile strain showing phenotypes breakdown and large, from early and later stages respectively, of the serial subculture treatment, were crossed to a wild-type $a$ strain. There was little difference between the progenies from the two crosses (table $6(a)$ ) in spite of the different phenotypes of one of the parents. Also the

TABLE 6

(a) Random spore progenies from the female-sterile strain showing phenotypes breakdown and large

\begin{tabular}{|c|c|c|c|c|c|}
\hline \multirow{2}{*}{ Parents } & \multicolumn{5}{|c|}{ Progeny } \\
\hline & $\mathrm{La}$ & $\mathrm{Sm}$ & $\mathrm{St}$ & $\mathrm{Bk}$ & Total \\
\hline Wild-type $a \times$ female-sterile $A$ (breakdown) & 59 & - & 9 & 10 & 78 \\
Wild-type $a \times$ female-sterile $A$ (large) & 68 & - & 3 & 6 & 77 \\
\hline
\end{tabular}

(b) The formerly female-sterile strain used as a female parent (random spores)

\begin{tabular}{|c|c|c|c|c|c|c|}
\hline \multirow{2}{*}{ Parents } & \multirow{2}{*}{$\begin{array}{l}\text { Genotype } \\
\text { of male } \\
\text { parents }\end{array}$} & \multicolumn{5}{|c|}{ Progeny } \\
\hline & & $\mathrm{La}$ & $\mathrm{Sm}^{\prime}$ & St & Bk & Total \\
\hline Female-sterile $A \times 5-7 a$. & $s b k$ & $3^{1}$ & I & 5 & 42 & 79 \\
\hline Female-sterile $A \times \mathrm{s}=3 a$. & $s+b k$ & 76 & - & I & - & 77 \\
\hline Female-sterile $A \times 4-3 a$. & $s^{+} b k^{+}$ & 74 & - & 3 & 3 & 80 \\
\hline
\end{tabular}


numbers of breakdown segregants in the two progenies were similar to those obtained from the original wild-type $\times$ female-sterile cross (table I). The development of phenotype large by the female-sterile strain did not therefore appear to involve genetic changes such as back mutation or the action of modifying genes. Neither of the progenies in table $6(a)$ showed any differentiation between the large and small phenotypes, which, because of the high ascospore viabilities of these crosses, can only mean a breakdown in the distinction between these phenotypes.

The female-sterile strain which developed protoperithecia as a result of the serial subculture treatment was used as a female parent in three crosses made by the spermatisation technique (table $6(b))$. The male parent of the first cross $5-7 a$ was genetically $s b k$ and the progeny contained large and breakdown cultures. The formerly female-sterile strain clearly passed on the ability to form large protoperithecia to some of the progeny cultures. The male parent of the second cross, I- $3 a$, was a large strain with genotype $s^{+} b k$, and the progeny was typical of that expected from an intercross of two large strains, apart from one culture which probably had genotype $s b k$. In the third cross the male parent 4-3a was a large strain with genotype $s^{+} b k^{+}$, and yet there were three breakdown cultures in the progeny. As 4-3a did not carry $b k$, these must have originated from the newly female-fertile strain. This supports the view that back mutation was not involved in the change from female-sterile to the large phenotype. The lack of small segregants in this progeny, and in those shown in table $6(a)$, also shows that the change in phenotype was not due to modifying genes. From these results, it appeared that the female-sterile strain had gained a non-genetically determined ability to form large protoperithecia in much the same manner as $s b k$ strains produced small protoperithecia.

\section{DISCUSSION}

\section{(i) The variable phenotypic expression of $s$ bk}

The most interesting of the four genotypes present in the progeny of the wild-type $\times$ female-sterile cross was the double mutant $s b k$, which varied in expression showing phenotypes ranging from different intensities of breakdown pigmentation through intermediate sterile forms to reduced protoperithecial production (small). This phenotypic variation was not caused by gene differences as could be seen from the presence of both phenotypes breakdown and small in some cultures, the two cultures of $s b k$ spore pairs showing different phenotypes, and breakdown ascospore cultures being genetically identical with their subcultures which developed phenotype small. Successive culture of the phenotypically small $s b k$ strains at $35^{\circ} \mathrm{C}$. for 24 hours, followed by maturation at $25^{\circ} \mathrm{C}$., readily induced breakdown to reappear. The appearance of breakdown in ascospore cultures or as a result of serial subculture only, suggests that small was the normal phenotype of $s b k$, and that breakdown was induced by the serial subculture and, to 
a lesser degree, by some feature of ascospore cultures. The increased intensity of breakdown with continuation of serial subculture, and its maintenance after the return to normal subculture conditions showed that its induction was not a direct environmental effect. Also, the induction of breakdown carried over to the progeny of the next sexual generation. Treatment of both parents of large $\times$ breakdown crosses gave a much increased expression of breakdown in the progeny, and, also, considerable inviability of $s b k$ ascospores. Such dauermodificationlike behaviour suggests an epigenetic system of a physiological or cytoplasmic nature was present, and that the different phenotypic expressions of the nuclear genotype $s b k$ were functions of different interchangeable, partly autonomous states of this system. The inviability of $s b k$ ascospores accompanying the strong expression of breakdown would appear to be an extreme manifestation of the cellular condition causing $s b k$ to be expressed as breakdown. Like breakdown, it occurred randomly and regardless of spore pairs; also, the intensification of breakdown pigmentation in the serial subculture series was accompanied by slowing down of hyphal growth and eventually death of the hyphæ.

The range of phenotypic expression with several distinct phenotypes shown by $s b k$, and the common reversion of the induced breakdown phenotype to small in subcultures, together with the immediate induction of breakdown by serial subculturing, make it unlikely that the appearance of breakdown was due to depletion and loss of controlling cytoplasmic elements. Rather, the change from one phenotype to another, and reversion to the original, would suggest that changes in the equilibrium of cytoplasmic elements were involved. Different equilibrium states of cytoplasmic elements could produce a continuous series of phenotypes, and would allow rapid changes and considerable stability at different times, depending on the factors controlling the equilibrium. The extreme cellular condition, characterised by reduced vitality of hyphæ and inviability of many $s b k$ ascospores, could result from an extreme change in the equilibrium by which one of the cytoplasmic elements was either greatly reduced or lost causing death of the cell. An alternative and perhaps more likely explanation of the extreme cellular condition is that the initially induced change in the cytoplasmic equilibrium caused hyphal breakdown, and that further change in the equilibrium increased this abnormal physiological condition so as to eventually cause death of the hyphæ. Ascospores with this extreme equilibrium state would have a high degree of physiological abnormality and would not germinate. This is a physiological limit to phenotypic variation as described by Jinks (1957), and has already received comment (Fitzgerald, I958).

Distinct cytoplasmic elements are not necessary to explain the phenotypic behaviour of $s b k$. An epigenetic system of gene interaction can do this. Between the initial action of a gene and its ultimate phenotypic effect, a wide field of gene interaction and possibly cytoplasmic influence intervenes. Normally a stable equilibrium of these 
interactions results in a definite canalisation which produces a stable phenotype. However, in some cases, especially with mutant genes, a less stable equilibrium might be expected. Small influences inside or from outside the cell would disturb such an equilibrium and lead to different canalisations from which a range of phenotypic effects might be expected. A gene concerned with such an epigenetic system would show variation in its expression, and furthermore, depending on the ability of a particular canalisation to maintain itself once established, examples of limited inheritance might be expected. Such a system could explain the changes in phenotypic expression of $s b k$ and the stability of the induced changes. It has similarities to the steady state system of Delbrück (see Ephrussi, r 953; Beale, I954).

Regarding the feature of ascospore cultures which induced breakdown, it was proposed that this might be the $60^{\circ} \mathrm{C}$. heat treatment for 50 minutes to initiate ascospore germination. However, this phenotype was present in ascospore cultures germinated by furfural instead of heat (Emerson, I 948); and heating subculture inocula to $60^{\circ} \mathrm{C}$. failed to induce breakdown.

The behaviour of $s b k$ has some similarities to the $n d$ and $w d$ mutants which initiated degenerative changes in Neurospora crassa (Sheng, I $95 \mathrm{I} a, b)$. Whereas external stimuli, in the form of ascospore activation or continued serial subculture, caused a slowing down of hyphal growth and eventually hyphal death of $s b k$ strains, death or degeneration of strains carrying the $n d$ and wd mutants occurred spontaneously within a few vegetative transfers. Sheng found that this progressive degeneration involved a cytoplasmic run-down or ageing, possibly due to the accumulation of a self-intoxicating substance in the growing frontier, the evidence being that replacement of the cytoplasm by making heterocaryons or crossing to wild-type temporarily reversed the degenerative phenotypic changes. While the tendency of the $s b k$ strains to overcome the adverse induced effects contrasted with the degenerative action in the $n d$ and $w d$ strains, it is likely that both types of behaviour were functions of similar cellular systems.

\section{(ii) The nature of the female-sterility}

Four different genotypes, each with corresponding phenotypes, were present in the progeny of the wild-type $\times$ female-sterile cross. The wild-type parent corresponded to phenotype large, and while the female-sterile parent was like the sterile phenotype, it retained its sterility over several subculture generations unlike the progeny cultures which were sterile on!, in ascospore cultures and developed small protoperithecia in subcultures. This failure to fully reproduce the female-sterility in the progeny suggested that it involved more than the gene differences shown to exist between the parent strains. However, the parental female-sterile strain was not completely stable, and as a result of serial subculture it developed large protoperithecia which were 
maintained subsequently and were fertile. The wild-type parent consistently produced large protoperithecia and therefore carried allele $s^{+}$, whereas the female-sterile parent presumably carried allele $s$. Serial subculture failed to show breakdown in the wild-type strain, but showed it in the female-sterile strain, indicating the presence of allele $b k$. This is supported by the demonstration of breakdown only in serial subcultures of large strains with the $s^{+} b k$ genotype. The development of phenotype large by the female-sterile strain did not involve back mutation of $s$, and was not likely due to mutation at other loci modifying $s$. The female-sterile strain was therefore genetically similar to the sterile strains with genotypes $s b k$, and like them could change to a fertile phenotype as a result of some change other than gene mutation. It differed from the sterile strains only in its more stable sterility, and in its development of phenotype large instead of small. It has already been shown that $s b k$ progeny cultures produced a sterile phenotype only when they were coupled with a particular non-permanent cellular state. Likewise, the female sterility of the original strain can be considered to have resulted from the association of $s b k$ with a particular cellular state belonging to the same or a similar system as that controlling the expression of $s b k$ in the progeny cultures. A likely hypothesis therefore is that the originally wild-type strain developed mutant genes $s$ and $b k$, which did not affect its female-fertility in the presence of a normal cellular state. An aberrant, semi-permanent cellular state then gradually became established in the mycelium as a result of continued vegetative reproduction, and possibly from ageing; and as it developed it caused a gradual diminishing of female-fertility, and finally complete failure to form protoperithecia.

Alternative factors interacting with $s b k$ to cause the female-sterility can be postulated. During the long period of asexual propagation the original female-sterile strain may have accumulated gene mutations and become heterocaryotic in respect of factors affecting the production of protoperithecia, not all of which were present in the progeny strains; or chromosome unbalance resulting from polysomy or structural hybridity may have arisen. A possible indication of such abnormalities were the four asci in the original wild-type $\times$ female-sterile progeny which showed abnormal growth and originated from different perithecia than did the other asci. However, the abnormal growth in all spores of these four asci indicated an overall extreme cytoplasmic modification of genotype expression rather than the effect of segregating genes, and does not support this view. Also, it is difficult to account for the phenotypic behaviour of the sterile parental strain by such genetic mechanisms. It is clear that chromosome unbalance, if it existed in the sterile strain, did not occur in its progeny because the irregular ratios obtained from crosses of progeny strains were not consistent with the ratios expected from chromosome unbalance in a haploid organism (see Emerson, 1956), but were caused by non-genetic variation in the expression of $s b k$. 


\section{SUMMARY}

I. Female-sterility, due to failure to form protoperithecia, was found in a biochemically wild-type strain of $\mathcal{N}$. crassa.

2. This sterile strain differed from wild-type by two genes. Genotypes $s^{+} b k^{+}$and $s^{+} b k$ were fully fertile wild-type, and $s b k^{+}$showed reduced protoperithecial development but was fertile. Genotype $s b k$ varied in expression with phenotypes ranging from a reduced femalefertile form to a sterile form producing dark pigmentation. This latter phenotype was accompanied by considerable ascospore inviability.

3. The phenotypic variation of $s b k$ was not due to gene differences but appeared to be determined by different equilibrium states of a cellular system intervening between $s b k$ and its phenotypic effects. Under certain conditions, some of these states were self-perpetuating.

4. The female-sterile strain had the $s b k$ genotype, and it appeared that sterility resulted when this genotype was associated with a particular cellular state belonging to the same or a similar system as that which controlled the expression of $s b k$ in the progeny cultures.

Acknowledgments: This work was done largely at the Genetics Department, University of Adelaide, on a New Zealand National Research Fellowship. I wish to express my thanks to Professor D. G. Catcheside, F.R.S., formerly of Adelaide, for his interest and helpful advice.

\section{REFERENCES}

BEALE, G. H. 1954. The genetics of Paramecium aurelia. University Press, Cambridge. CATCHeside, D. G. 1959. Cytoplasmic inheritance. Nature, 184, 1012-1015. DODGE, B. O. 1946. Self-sterility in bisexual heterocaryons of Neurospora. Bull. Torr. Bot. Club, 75, 410-416.

EMERSON, M. R. 1948. Chemical activation of ascospore germination in Neurospora crassa. 7. Bact., 55, 327-330.

EMERson, s. 1956. Notes on the identification of different causes of aberrant tetrad ratios in Saccharomyces. C.r. Lab. Carlsberg. Sér. physiol., 26, 7 I-86.

EPHRUSSI, B. 1953. Nucleo-cytoplasmic relations in micro-organisms. Clarendon Press, Oxford.

FitZGerald, P. H. 1958. A case of limitation of phenotypic variation. Nature, 181,436 . HIRSCH, H. M. 1954. Environmental factors influencing the determination of protoperithecia, and their relation to tyrosinase and melanin formation in Neurospora crassa. Physiol. Plant., 7, 72-97.

JINKs, J. L. 1954. Somatic selection in fungi. Nature, 174, 408-410.

JiNks, J. L. 1956. Naturally occurring cytoplasmic changes in fungi. C.r. Lab. Carlsberg Sér. physiol., 26, $183-203$.

Jinks, J. L. 1957. Selection for cytoplasmic differences. Proc. Roy. Soc. B., 146, 527-540. NANNey, D. L. 1958. Epigenetic control systems. Proc. Natl. Acad. Sci. (U.S.), 44. $712-717$.

SHENG, T. C. 1951a. A gene that causes natural death in Neurospora crassa. Genetics, $36,199-212$.

SHENG, T. C. 1951 $b$. The genetic basis of woolly degeneration in Neurospora crassa. Bot. Gaz., II3, 203-206.

WESTERGAARD, M., AND HIRSCH, H. M. 1954. Environmental and genetic control of differentiation in Neurospora. Recent Developments in Cell Physiology. Colston Papers, 7, $171-183$.

WESTERGAARD, M., AND MITCHeLl, H. K. 1947. Neurospora. V. A synthetic medium favouring sexual reproduction. Am. F. Bot., 34, 573-577. 